\title{
Emulating Bearing Faults - A Novel Approach
}

\author{
Bram Corne, Jos Knockaert and Jan Desmet
}

\begin{abstract}
The relation between evolving mechanical faults in rotating electrical machines and their reflection in the machines' electrical parameters still requires a lot of research. This implies serious obstructions in the evolution of e.g. Motor Current Signature Analysis as a complete and reliable condition monitoring technology. This paper presents the translation of common bearing faults into specific rotor-stator movements using finite element modeling. Subsequently, a novel method to elucidate the complex relation between rotor movements and the electrical parameters of an induction machine using an experimental test setup is described, dimensioned and simulated. Replacing one of the induction machine's bearings with an Active Magnetic Bearing will give the opportunity to create specific rotor movements and consequently evaluate different programmable mechanical faults and their reflection in the stator current and/or voltage with high relevance and reproducibility.
\end{abstract}

Index Terms-MCSA, Condition monitoring, Fault diagnosis, Induction motors, Emulation, Magnetic levitation

\section{INTRODUCTION}

If a condition monitoring technology for rotating electrical machinery elaborates, validation is a crucial step in the development. Many authors have been searching for an easy way to implement quantified mechanical faults in electrical machines on an academic basis with reproducibility and relevance to industrial applications (mainly bearing related) [1]-[9]. For now, e.g. emulating single point bearing faults in electrical machines remains difficult, time-consuming and imprecise. The widely used method is replacing one of the bearings of the Device Under Test (DUT) by a bearing with an artificial implemented fault [1]-[9]. Subsequently, the condition related parameters (e.g. temperature, vibration, stator current...) could be analyzed and evaluated with respect to the healthy condition. Unfortunately, the reproducibility is already lost by remounting the bearing because the total systems characteristics like stiffness and damping are highly depending on the bearings mounting. Additionally, the way faults are enforced on the bearing is often very different with respect to the real situation, which highly decreases the relevance of the test-results. For example mechanical outer race pitting is usually induced by drilling holes of different sizes in the outer race, which should represent several severity stages [1], [2], [4], [7], [8]. Unfortunately, those holes are hardly relevant to real evolving mechanical pitting [5], [10], [11].

As the core research of the authors is the development of detecting mechanical faults in electrical machines by analyzing the stator current, building an accurate mechanical fault emulator is crucial. Research already concluded that the stator current can be used as a tool to examine the mechanical

B. Corne, J. Knockaert and J. Desmet are with the research group Lemcko, Department of Electrical Energy, Systems and Automation (EESA), Faculty of Engineering and Architecture (FEA), Ghent University; Graaf Karel de Goedelaan 5, B-8500 Kortrijk, Belgium; email: bram.corne@ugent.be condition of the machine, but stating the severity-stage of detected faults is still lacking [12]. When the mechanical fault emulator is build, finding relations between the fault severity and the stator current fault signature under different environmental influences should be feasible. This paper focuses on the specific rotor movement when bearing faults occur and the accurate emulation of those faults on an $11 \mathrm{~kW}$ Induction Machine (IM) using an Active Magnetic Bearing (AMB). It must be noticed that in this paper, a mechanical fault is defined as a situation created with mechanical root cause leading directly or indirectly to the premature failure of an IM. Three sorts of bearing faults will be discussed: outer race, inner race and cage faults.

\section{Concept}

All kinds of mechanical faults that can occur in an IM can be related to specific radial movements of the rotor in relation to the stator (bearing faults, rotor unbalance, misalignment). Therefore, emulating a mechanical fault in an electrical machine could be done by controlling the position of the rotor in relation to the stator with high accuracy. To do so, replacing one of the mechanical bearings of the machine by an AMB can fulfill the fault-related rotor-stator movements. When specific rotor movements for certain faults and their severity are obtained (based on simulations, analytical calculations or measurements), the outcome can serve as set points for the magnetic bearing's control system. The AMB has the major advantage that stiffness and damping characteristics of a mechanical bearing can be implemented in the control loop. Consequently, all the machines' system characteristics can be held constant while the mechanical faults are emulated with an accessible user interface. The operating machine does not have to be interrupted or shut down to imply different or evolving faults. Therefore, the relation between the severity of a specific fault and the reflection in the stator current can easily be investigated thoroughly under different circumstances gathering torque, speed, temperature, supplied voltage... This way of emulating faulty conditions results in the achievement of high quality in both relevance and reproducibility.

In §II, the relation between different mechanical faults and their implied rotor movement is elucidated for bearing outer race, inner race and cage faults. Those obtained results will serve as set points for the AMB system, which is constructively dimensioned in §III focusing on the steady state and dynamic requirements. Subsequently, the specifications for the control system are obtained. Conclusions regarding this paper and a view on future research can be found in §IV. 


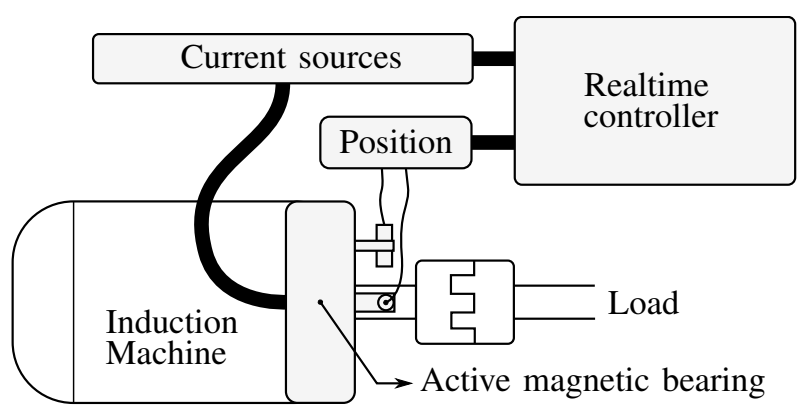

Figure 1: Schematic concept of using an AMB as a mechanical fault emulator

\section{ROTOR MOVEMENTS WITH FAULTY CONDITIONS}

As previously mentioned, the intention of the fault emulating test-rig is manipulating the rotor's position with respect to the position of the stator. As an input for the test-rig, crucial information is needed about rotor movements under different faulty conditions. It is a challenge in determining those movements for all bearing faults gathering severity conditions, rotor speed, fault location, mechanical load, bearing parameters... However, when the set points are determined, the emulation of the fault will be very similar to the real situation, because the implemented AMB excites the rotor on exactly the same place as the mechanical bearing would do.

Calculating rotor movements under faulty bearing conditions, mechanical Finite Element Modeling (FEM) is done using the software Siemens NX - PLM. The package is used to simulate the rotor movement when an outer race bearing fault occurs and to extract the bearing system characteristics, which are compared with the properties provided by the bearing supplier. Those system characteristics are used to approach the mechanical rotor-stator relation as a simplified mass-springdamper system. That simple model can be excited analytically with the use of fault-related impulse functions, representing the rotor being excited by a bearing fault. The resulting system responses serve as set points for the AMB. The use of impulse functions is necessary to calculate the rotor movements for inner race bearing faults accurately. Additionally, the calculation-time of obtaining the rotor movements by using the spring damper system is much less than executing FEM for every fault.

\section{A. Bearing outer race fault}

To obtain a good estimation of the rotor movements in relation to the stator when an outer race fault occurs, a bearing ball is modeled in FEM which rolls over a pit of $1 \mathrm{~mm}$ long and $100 \mu \mathrm{m}$ deep (Figure 2). The depth of the pit is a realistic starting point. The increase in severity will be a change in length, not in depth [5], [10]. Simulating the entire bearing system (outer race, inner race, cage, balls, rotor, stator...) is currently not possible due to the limitations of the used FEMsoftware package. Simplifications of the system where carried out.

The outer race and the inner race are represented by two solid profiles which are able to translate with respect to each

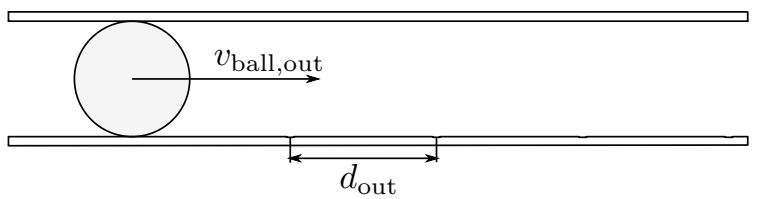

Figure 2: Siemens NX draft simulation of pitting in the bearing's outer race

other. Between those profiles, only one bearing ball is drawn which will move due to an implied translation and rotation. Several pits are added in order to simulate the other bearing balls rolling over the same pit. The specified distance between the pits is in fact the distance between the bearing ball contact points with the outer race (Figure 3 ). This distance $d_{\text {out }}$ can be expressed as:

$$
d_{\mathrm{out}}=\frac{\pi}{m}(p d+b d \cdot \cos \beta)
$$

With:

$\begin{array}{ll}d_{\text {out }} & \text { distance between pits in simulation }[\mathrm{m}] \\ m & \text { number of bearing balls }(8) ; \\ p d & \text { pitch diameter }(74 \mathrm{~mm}) ; \\ b d & \text { ball diameter }(17.5 \mathrm{~mm}) ; \\ \beta & \text { contact angle }\left(0^{\circ}\right) .\end{array}$

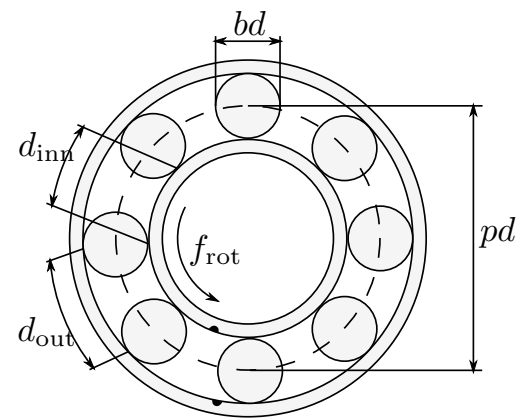

Figure 3: Graphical presentation of the used bearing parameters

The bearing ball has the dimensions and the mass of the original IM's replaced bearing used in the test-rig, superposed with the mass corresponding to the gravitational force which was working on that IM's bearing at DE $\left(m_{\text {rot,DE }}\right)$. This implies that all inertia properties of the ball in every direction remain the same except the inertia in the vertical direction (perpendicular on the direction of movement), which is now related to $m_{\text {rot,DE}}$. The angular velocity in which the bearing balls are rolling over the pit is equal to the Fundamental Train Frequency (FTF) and can be written as:

$$
f_{\mathrm{FTF}}=\frac{f_{\mathrm{rot}}}{2}\left(1-\frac{b d}{p d} \cos \beta\right)
$$

This results in the following velocity of the moving bearing ball in reference to the outer race:

$$
v_{\text {ball, out }}=\pi(p d+b d \cdot \cos \beta) f_{\mathrm{FTF}}
$$

With the parameters $d_{\text {out }}$ and $v_{\text {ball,out }}$, the faulty bearing can be simulated in the mechanical FEM software. Out of 
the simulations, the vertical response in displacement and acceleration are extracted and presented for one specific outer race bearing fault in respectively Figure 4 and Figure 5. Out of Figure 5, the proportional force that needs to be applied by the AMB (to create the corresponding movement) as a function of time is obtained, which already briefly indicates the dynamic requirements of the AMB.

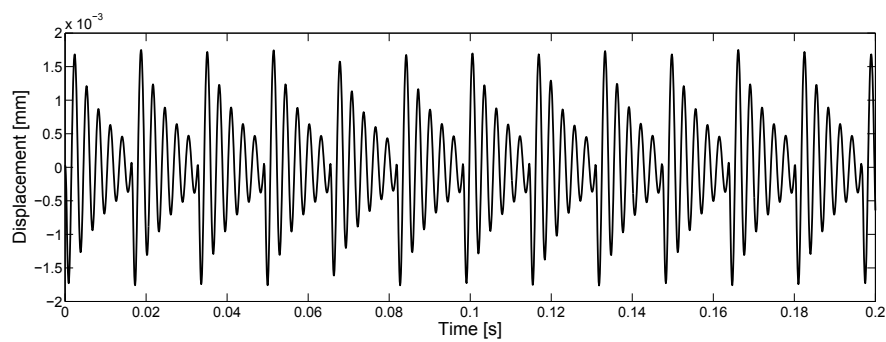

Figure 4: Vertical rotor displacement of simulated bearing outer race fault

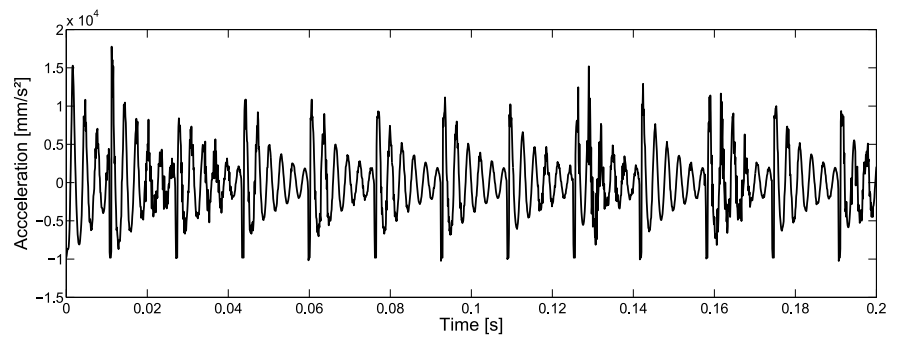

Figure 5: Vertical rotor acceleration of simulated bearing outer race fault

Out of the FEM presented in Figure 4, some interesting system properties can be extracted in order to simplify prospective calculations. The mechanical bearing can be simplified as a Single Degree Of Freedom (SDOF) system with a certain stiffness $k_{\mathrm{rad}, \mathrm{DE}}$, mass $m_{\mathrm{rot}, \mathrm{DE}}$ and damping $c_{\mathrm{rad}, \mathrm{DE}}$ following the force equation:

$$
m_{\mathrm{rot}, \mathrm{DE}} \cdot \ddot{y}(t)+c_{\mathrm{rad}, \mathrm{DE}} \cdot \dot{y}(t)+k_{\mathrm{rad}, \mathrm{DE}} \cdot y(t)=F(t)
$$

$y(t)$ represents the instantaneous vertical displacement of the rotor with respect to the stator at time $t . F(t)$ represents the force between the rotor and the stator (the force acting on the bearing). $\ddot{y}(t)$ and $\dot{y}(t)$ are respectively the instantaneous acceleration and velocity of the rotor with respect to the stator in the vertical direction.

Out of the response function of the system (presented in Figure 4), the system parameters can be obtained with the use of:

$$
\delta=\frac{1}{n} \ln \left(\frac{y(t)}{y(t+n \cdot T)}\right)
$$

$\delta$ is directly derived from Figure 4 , based on the peak magnitudes $y(t)$ and $y(t+n \cdot T)$ with $n$ the number of periods $T$. This leads to the calculation of the proportional damping factor $\zeta$ :

$$
\zeta=\frac{1}{\sqrt{1+\left(\frac{2 \cdot \pi}{\delta}\right)^{2}}}
$$

The natural frequency, $\omega_{\mathrm{d}}$, is also directly derived out of Figure 4 because $\omega_{\mathrm{d}}=\frac{1}{T}$. With the known equivalent rotor mass at $\mathrm{DE}, m_{\mathrm{rot}, \mathrm{DE}}$, the radial stiffness at DE, $k_{\mathrm{rad}, \mathrm{DE}}$, is obtained by applying:

$$
k_{\mathrm{rad}, \mathrm{DE}}=m_{\mathrm{rot}, \mathrm{DE}} \cdot \omega_{\mathrm{d}}^{2}
$$

Subsequently, the radial damping at DE, $c_{\mathrm{rad}, \mathrm{DE}}$, is obtained using:

$$
c_{\mathrm{rad}, \mathrm{DE}}=2 \cdot \zeta \cdot m_{\mathrm{rot}, \mathrm{DE}} \cdot \omega_{\mathrm{d}}
$$

With $m_{\text {rot,DE}}, k_{\text {rad,DE }}$ and $c_{\text {rad,DE, the whole rotor/stator }}$ system is characterized by (4). The calculated stiffness and damping deviate $\sim 12 \%$ of the corresponding parameters provided by the bearing supplier.

For further and future calculations, the force impulse function related to an outer race bearing fault can be applied on the simplified bearing system with the displacement response function as a result, which serves as set point for the AMB. The force impulse functions can be established analytically or via FEM for different shapes of pits, rotational speeds, external forces and bearing parameters.

\section{B. Bearing inner race fault}

A bearing inner race fault seems in principle very similar to an outer race fault. Nevertheless, the fault propagation is to complex to simulate with the mechanical FEM software. With the use of previously derived system parameters of the SDOF interaction between the stator and the rotor, the vertical rotor movements can be obtained analytically. The velocity of the inner ring with respect to the ball velocity is:

$$
v_{\text {ball,in }}=\pi(p d-b d \cdot \cos \beta)\left(f_{\text {rot }}-f_{\mathrm{FTF}}\right)
$$

The relative distance, $d_{\text {inn }}$, between the different contact points of the balls with the inner race (Figure 3) can be expressed as:

$$
d_{\text {inn }}=\frac{\pi}{m}(p d-b d \cdot \cos \beta)
$$

With the use of $v_{\text {ball,inn }}$ and $d_{\text {inn }}$, the pulse train frequency, $\frac{v_{\text {ball,inn }}}{d_{\text {inn }}}$, is calculated, presented in Figure 6. The magnitude of the impulse function $(450 \mathrm{~N})$ is based on the reversely calculated impulse function of the previously modeled outer race bearing fault. The impulse function of that outer race fault is mathematically derived by performing the deconvolution of the response function (Figure 4) and the impulse response function (analytically obtained with the system parameters $m_{\text {rot,DE }}, k_{\text {rad,DE }}$ and $\left.c_{\text {rad,DE }}\right)$.

Due to the fault rotating in the machine, the rotor is not always vertically exited when a bearing ball passes the pit. The relative force interaction between the vertical rotor direction and a single point on the inner race is illustrated in Figure 7. The interaction function describes a positive alternation of a unity sine function with a frequency $f_{\text {rot }}$. The multiplication of the pulse train and the interaction curve results in Figure 8 , which represents the impulse force of the inner race fault on the vertical movement of the rotor. Due to the critical role of the interaction curve in the faulty inner race response calculation, the rotor movements could not be derived with the FEM, but has to be analytically determined with the use of the simplified SDOF model. 


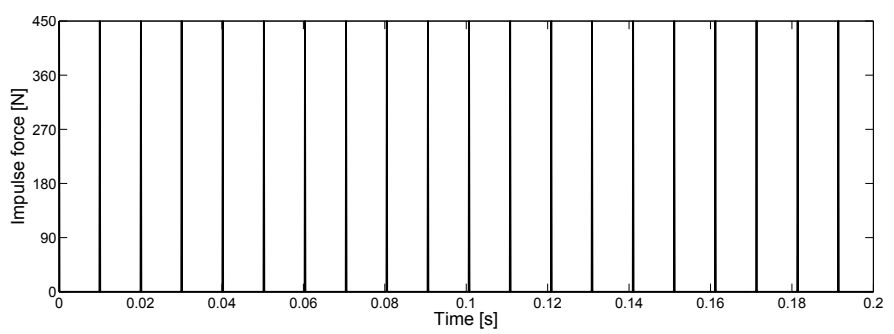

Figure 6: The pulse train of the bearing balls passing the inner race fault

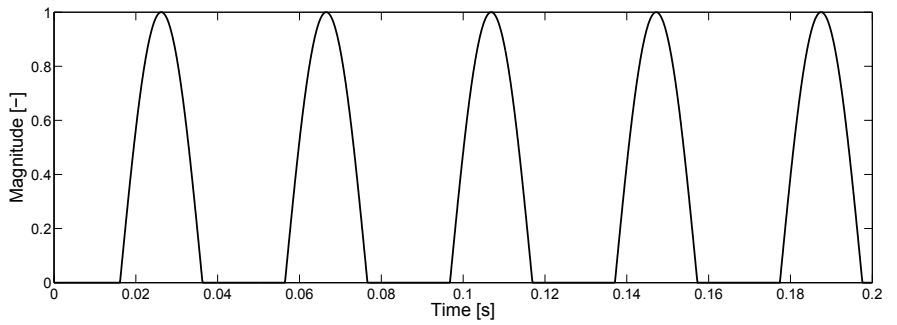

Figure 7: The interaction between a single inner race point and the vertical rotor position

The system response function obtained with the impulse function out of Figure 8 and the system parameters $m_{\text {rot,DE, }}$ $k_{\text {rad,DE }}$ and $c_{\text {rad,DE }}$ is presented in Figure 9. This way of obtaining the AMB set points is less time-consuming than FEM and will be used for the calculation of both inner race and outer race bearing fault rotor movements. However, FEM can still be used to determine the exact pulse duration and magnitude of the impulse functions for different severity stages of the pits.

\section{Bearing cage fault}

When a bearing cage fault occurs, the bearing balls are not equally divided around the inner ring (Figure 10). Sequentially, the rotor is subjected to a dynamic eccentricity at a frequency equal to the FTF $\left(f_{\mathrm{FTF}}\right)$. Therefore, the equivalent rotor displacement $y(t)$ and $x(t)$ can be expressed as:

$$
\begin{aligned}
& y(t)=A_{\text {cage }} \cdot \sin \left(2 \cdot \pi \cdot f_{\mathrm{FTF}} \cdot t\right) \\
& x(t)=A_{\text {cage }} \cdot \sin \left(2 \cdot \pi \cdot f_{\mathrm{FTF}} \cdot t+\frac{\pi}{2}\right)
\end{aligned}
$$

The magnitude $A_{\text {cage }}$ in the expression can be used as an indicator for the fault severity. Because the bearing cage fault set point vary relatively slow in time with respect to an outer race bearing fault $\left(f_{\mathrm{FTF}} \simeq 7 \mathrm{~Hz}\right.$ for the DUT at a rotational speed of $1460 \mathrm{rpm}$ ), the focus in dimensioning the control system of the AMB will lie on emulating a bearing outer race fault.

\section{MAGNetiC BEARING AS A ROTOR EXCITER}

\section{A. $A M B$ dimensions}

Many research has already been done on AMBs, but with totally different applications and scopes. An AMB is in general applied to reduce the losses of the machine by decreasing the losses of the machine's bearings (shifting the losses from

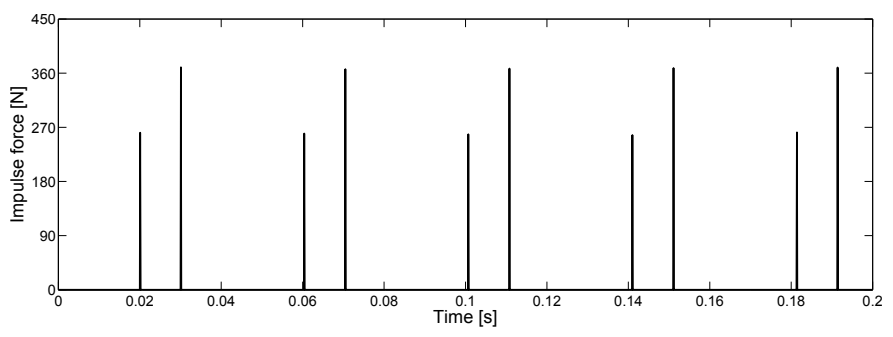

Figure 8: The impact of an inner race fault on the vertical rotor position

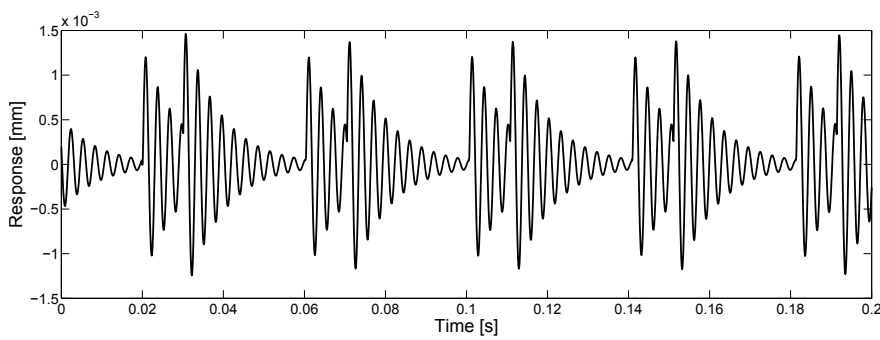

Figure 9: The vertical rotor movement when a bearing inner race fault occurs

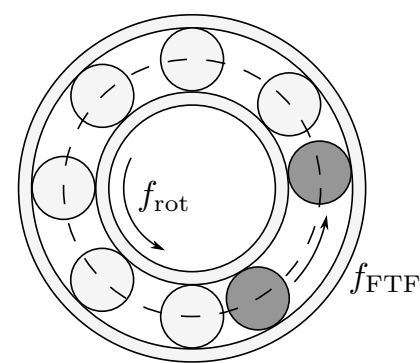

Figure 10: Rotor dynamic eccentricity when bearing cage fault occurs

friction losses to copper and iron losses) [13]-[16]. Therefore, AMBs are more applicable for high-speed machines. In this application, an AMB is used in order to emulate mechanical faults in an IM. Thereby, the AMB's effectiveness is more important than the efficiency. Consequently, loss calculations are excluded for this publication.

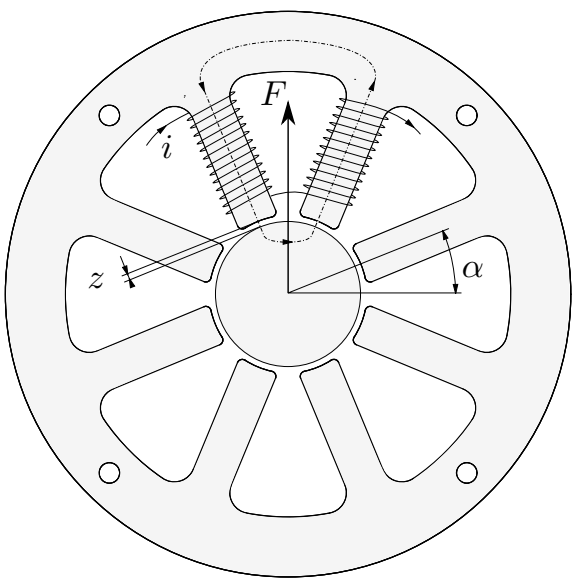

Figure 11: Magnetic bearing topology 
Firstly, the AMB must take over the functionalities of the mechanical bearing. The forces imposed on the rotor by the bearing will be depending on the gravitational force $(\sim 215 \mathrm{~N})$, the Unbalanced Magnetic Pull (UMP) $(\sim 500 \mathrm{~N}$ for $11 \mathrm{~kW}$, [17], [18]) and the requested rotor dynamics and strength of the AMB (proportionally obtained in Figure 5). This AMB operation range will be taken into account in the steady state dimensioning, following (13).

Secondly, to achieve the research goal, the rotor will be constantly moving relatively to the stator. Therefore, the AMB must be dimensioned with an unusual large air gap between the AMB and the rotor-shaft $(2 \mathrm{~mm})$ in order to maintain the freedom of the rotor moving around in the entire air gap of the DUT $(0.85 \mathrm{~mm})$. The physical topology used in this application could be found in Figure 11.

The attractive force between the AMB and the rotor-shaft generated by the AMB is [14], [19]:

$$
F(t)=\mu_{0}\left(\frac{N \cdot i(t)}{\frac{l_{\text {stat }}}{\mu_{\mathrm{r}_{\text {stat }}}}+\frac{l_{\text {sh }}}{\mu_{\mathrm{r}_{\mathrm{sh}}}}+2 \cdot z(t)}\right)^{2} A_{\mathrm{a}} \cdot \cos \alpha
$$

With:

$$
\begin{array}{ll}
F & \text { resulting force }[\mathrm{N}] ; \\
\mu_{0} & \text { permeability of vacuum }[\mathrm{H} / \mathrm{m}] ; \\
N & \text { number of turns in winding [-]; } \\
i & \text { current through the winding }[\mathrm{A}] ; \\
l_{\text {stat }} & \text { length flux path through transformer steel }[\mathrm{m}] ; \\
l_{\mathrm{sh}} & \text { length flux path through shaft }[\mathrm{m}] ; \\
\mu_{\mathrm{r}_{\mathrm{stat}}} & \text { relative permeability transformer steel }[-] ; \\
\mu_{\mathrm{r}} & \text { relative permeability shaft }[-] ; \\
z & \text { air gap between pole and shaft }[\mathrm{m}] ; \\
A_{\mathrm{a}} & \text { cross section surface of one pole }\left[\mathrm{m}^{2}\right] ; \\
\alpha & \text { angle between force and one pole }\left[{ }^{\circ}\right] .
\end{array}
$$

Some of the AMB parameters are fixed due to geometric or physical constraints such as material properties, construction space, control system properties... By changing the variable parameters such as number of windings, cross pole surface..., the most optimal AMB can be dimensioned with respect to the required domain of the generated force. With the optimal chosen parameters, Figure 12 is established which presents the steady state force as a function of the current through a coil of the AMB and the distance between the actuated pole and the rotor-shaft (air gap $z$ ). The flat yellow area in the figure $(\sim 2143 \mathrm{~N})$ is where the flux-density in the poles is maximal (magnetic material saturation at $\sim 1.8 \mathrm{~T}$ ).

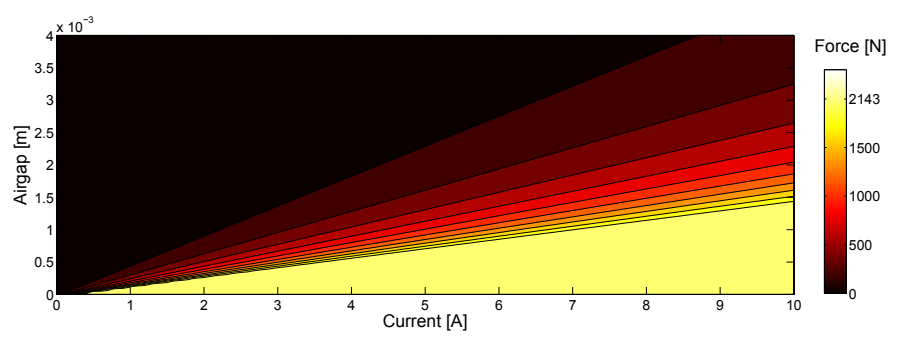

Figure 12: Steady state force of AMB

The $75 \mathrm{~mm}$ thick AMB is laminated with 75 sheets of $1 \mathrm{~mm}$ transformer steel. This reduces the eddy currents induced by the alternating flux, invoked by the switching DC and therefore the heating in the bearing. The free space between two poles is dimensioned based on the space needed for 200 turns of $0.8 \mathrm{~mm}^{2}$ varnished copper with $75 \%$ over-dimensioning.

\section{B. AMB dynamics}

Figure 12 indicates that the force created by the bearing should be sufficient to encounter the gravity force, compensate the UMP and dynamically move the rotor following a faulty bearing set point. As the AMB has electrically a resistiveinductive nature, the current change and furthermore the rotor displacement will lag the voltage change. Therefore, the dynamics between the rotor movements and the applied coil voltage must be investigated thoroughly. Notice that the rotor displacement is expressed as the air gap variation $z(t)$. This because the following expressions will hold for all four coils in the two directions of movements $(y(t)$ and $x(t))$.

The voltage applied on the coil $v(t)$ of the AMB can be described as a function of the current through the coil $i(t)$ and the air gap variation $z(t),[19]$ :

$$
v(t)=R_{\mathrm{w}} \cdot i(t)+L_{\mathrm{w}}(t) \frac{\mathrm{d} i(t)}{\mathrm{d} t}+K_{\mathrm{i}} \cdot \frac{\mathrm{d} z(t)}{\mathrm{d} t}
$$

With:

$$
\begin{aligned}
i(t) & \leq i_{\max } & & \\
R_{\mathrm{w}} & =\frac{\rho \cdot l_{\mathrm{w}}}{A_{\mathrm{w}}} & & \text { (winding resistance) } \\
L_{\mathrm{w}}(t) & =\frac{N^{2}}{\mathcal{R}_{\mathrm{m}}(t)} & & \text { (winding inductance) } \\
K_{\mathrm{i}} & =\left.\frac{\partial F}{\partial i}\right|_{i^{*}, z^{*}} & & \text { (current stiffness) }
\end{aligned}
$$

And the flux path reluctance is expressed as:

$$
\mathcal{R}_{\mathrm{m}}(t)=\frac{l_{\text {stat }}}{\mu_{\mathrm{r}_{\text {stat }}} \cdot \mu_{0} \cdot A_{\mathrm{a}}}+\frac{2 \cdot z(t)}{\mu_{0} \cdot A_{\mathrm{a}}}+\frac{l_{\mathrm{sh}}}{\mu_{\mathrm{r}_{\mathrm{sh}}} \cdot \mu_{0} \cdot A_{\mathrm{a}}}
$$

Following the topology presented in Figure 11, two poles are working as one force generator. Therefore, the winding is divided between the two poles (two times 200 turns) and connected to the current sources separately. This implies a reduction of the winding inductance $L_{\mathrm{w}}$ with a factor $4,(17)$. Following (14), the time constant of the electric system will be much lower, giving a more dynamic system response. It must be noticed that the coil inductance is already relatively low $(\sim 11 \mathrm{mH})$, because of the high reluctant flux-path due to the large air gap between the AMB and the rotor-shaft.

The relation between the current and the force is quadratic (13), which implies an inert second-order system between voltage and force. Furthermore, this calculated force does not imply a displacement instantaneously. The rotor moves according to the generated force $F(t)$ following:

$$
y(t)=\iint \frac{F(t)}{m_{\mathrm{rot}, \mathrm{DE}}} \mathrm{d} t
$$

Additionally, the air gap $(z(t) \sim y(t), x(t))$ and subsequently the coil inductance changes constantly. To ensure that the $\mathrm{AMB}$ is able to emulate accurately bearing faults in an IM, 
this complex relation is simulated in the next section in order to optimize the control system and the performance of the AMB.

\section{Control system}

The system response when an outer race bearing fault occurs, presented in Figure 4 is used to design and optimize the AMB's control system. To simulate the physical AMB system and the control loop, Matlab - Simulink is used. The entire physical AMB-system (electric and mechanic) is modeled. The AMB-model input is the supplied voltage on the upper, lower, left and right coils. The output is the rotor position in a 2D plain $(y(t)$ and $x(t))$. The AMB-model contains a combination of the formulas (13) and (14). As actuators for the model, hardchop full H-bridge current sources are used and simulated, which supply the voltage on the physical system based on the difference between the set point for the current and the real current through the coils. Set points for current are outputs of the PID-controllers (one for the vertical and one for the horizontal control), designed following [20]. The input of the PID-controller is the error between the real position and the set point for position (containing the bearing fault, Figure 4). To compensate the gravity force, a feed forward is implemented on the vertical control system which calculates the current based on the weight of the rotor and the corresponding air gap. On the horizontal control system, a constant bias force of $30 \mathrm{~N}$ is added on both right and left side to increase the stability of the system.

In the simulation, which is presented in Figure 13, the displacement function of Figure 4 is used as a time table set point for the control system. The rotor is as starting point virtually placed on the bottom of the magnetic bearing, in an idle position. Out of the results, the simulation states that the rotor is centered while starting up the system within $35 \mathrm{~ms}$. A more detailed view of the set point and the resulting response is illustrated in Figure 14

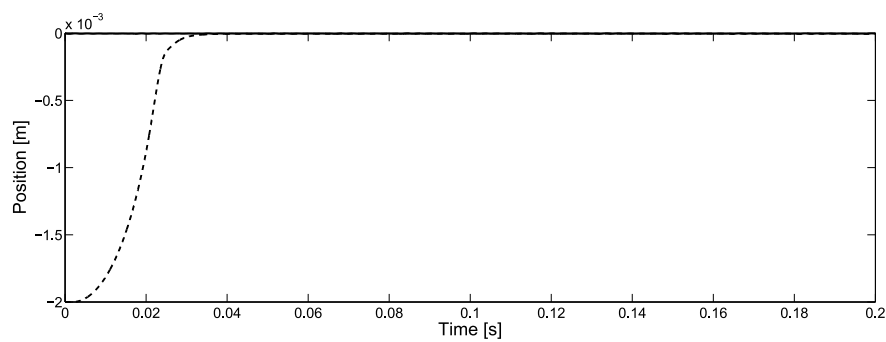

Figure 13: Position of the rotor (dashed line) and the set point (full line) of simulation results

Out of the AMB-model, currents through every coil of the magnetic bearing can easily be extracted. The current in the upper coil and the lower coil are presented in respectively Figure 15 and Figure 16. Figure 15 proves that the AMB is constructively well dimensioned because the current needed to emulate the bearing fault does not exceed the previously determined force/current range. It must be noticed that the dynamics of that current is fully depending on the DC-voltage supplied on the H-bridge current sources. If the current sources

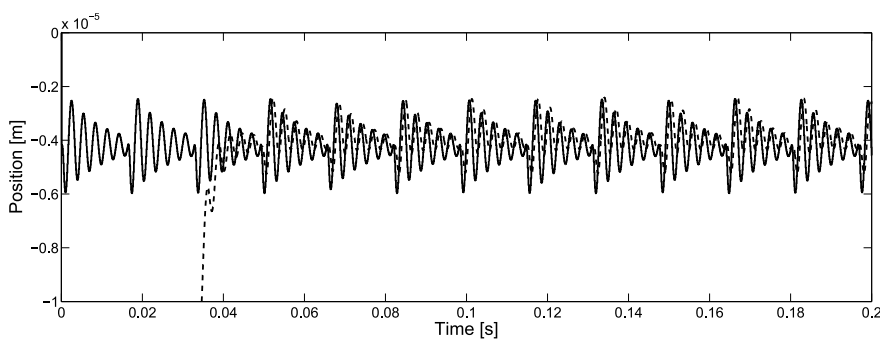

Figure 14: Detail on the position of the rotor (dashed line) and the set point (full line) of simulation results

are not able to follow the set points of the PID-controllers, the DC-link voltage can be increased. In this simulation a voltage of $60 \mathrm{~V}$ is used (which is low due to the small coil inductance).

The simulation results concluded that the magnetic bearing will easily emulate a very small specific bearing fault with the dimensions described in §III-A. With the AMB-model, future simulations can easily be performed with the focus on compensating mechanical unbalance, unbalanced magnetic pull, disturbance on the position measurement, torque-ripples...

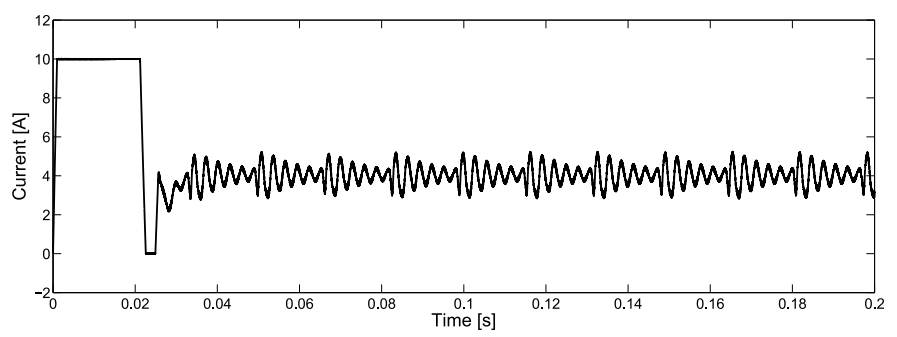

Figure 15: Current through the upper coil of the magnetic bearing during the bearing fault simulation

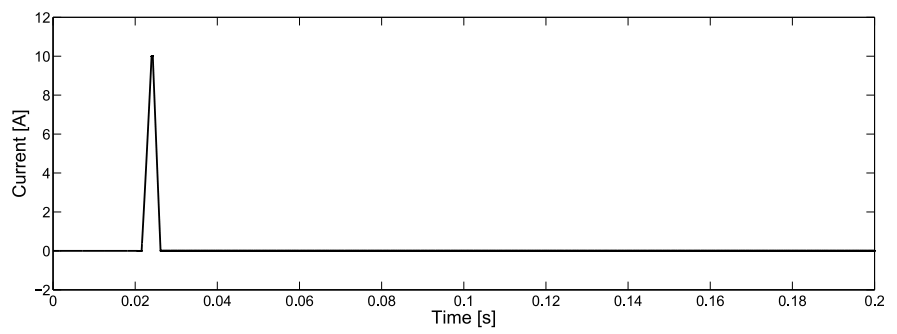

Figure 16: Current through the lower coil of the magnetic bearing during the bearing fault simulation

\section{CONCLUSION}

This paper presented initially an easy way to calculate specific rotor movements with respect to the stator with different faulty bearing conditions. This is obtained by applying force impulse function on a SDOF-model, characterized with the bearing system characteristics. Based on those displacement response functions, an active magnetic bearing is dimensioned in order to create those specific rotor movements on an $11 \mathrm{~kW}$ induction machine. The simulation model of the magnetic bearing is designed in Matlab - Simulink to optimize the PID control loop parameters. The simulation results indicate that the AMB will be able to emulate bearing faults accurately. 
For now, the AMB is already build and implemented into the IM (Figure 17). Currently, first tests have already been performed with rotational speeds close to $1500 \mathrm{rpm}$ with the focus on compensating the mechanical unbalance and torque variations due to the coupling with the load machine. In the near future, bearing faults will be emulated and the reflection of those faults in the stator current will be investigated thoroughly.

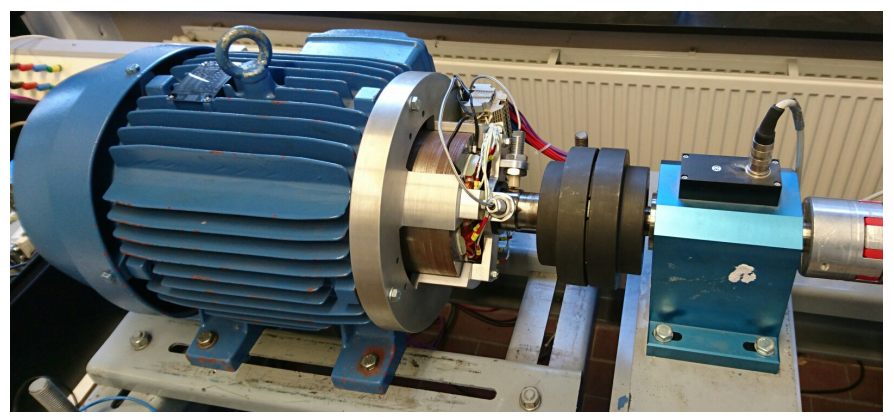

Figure 17: The AMB implemented at DE of the $11 \mathrm{~kW}$ IM

\section{REFERENCES}

[1] R. R. Schoen, T. G. Habetler, F. Kamran, and R. G. Bartheld, "Motor bearing damage detection using stator current monitoring," IEEE Transactions on Industry Applications, vol. 31, no. 6, pp. 1274-1279, 1995.

[2] L. Eren and M. J. Devaney, "Bearing Damage Detection via Wavelet Packet Decomposition of the Stator Current," IEEE Transactions on Instrumentation and Measurement, vol. 53, no. 2, pp. 431-436, 2004.

[3] M. Delgado, G. Cirrincione, a. Garcia, J. a. Ortega, and H. Henao, "A novel condition monitoring scheme for bearing faults based on Curvilinear Component Analysis and hierarchical neural networks," Proceedings - 2012 20th International Conference on Electrical Machines, ICEM 2012, pp. 2472-2478, 2012.

[4] W. Saadaoui and K. Jelassi, "Induction motor bearing damage detection using stator current analysis," 2011 International Conference on Power Engineering, Energy and Electrical Drives, no. May, pp. 1-6, 2011.

[5] W. Z. W. Zhou, B. L. B. Lu, T. Habetler, and R. Harley, "Incipien Bearing Fault Detection via Motor Stator Current Noise Cancellation Using Wiener Filter,' IEEE Transactions on Industry Applications, vol. 45, no. 4, pp. 1309-1317, 2009.

[6] S. McInerny and Y. Dai, "Basic vibration signal processing for bearing fault detection," Education, IEEE Transactions on, vol. 46, no. 1, pp. 149-156, 2003.

[7] M. J. Devaney and L. Eren, "Detecting motor bearing faults: Monitoring an induction motor's current and detecting bearing failure," IEEE Instrumentation and Measurement Magazine, vol. 7, no. December 2004 pp. 30-50, 2004.

[8] L. Frosini and E. Bassi, "Stator current and motor efficiency as indicators for different types of bearing faults in induction motors," IEEE Transactions on Industrial Electronics, vol. 57, no. 1, pp. 244-251, jan 2010.

[9] B. Corne, C. Debruyne, P. De Baets, and J. Desmet, "Stator current measurements as a condition monitoring technology - The-state-of-theart," in 2014 International Conference on Electrical Machines (ICEM). IEEE, sep 2014, pp. 1659-1665

[10] J. R. Stack, T. G. Habeter, and R. G. Harley, "Fault classification and fault signature production for rolling element bearings in electric machines," IEEE Transactions on Industry Applications, vol. 40, no. 3, pp. 735-739, 2004.

[11] D. S. Shah and V. N. Patel, "A Review of Dynamic Modeling and Fault Identifications Methods for Rolling Element Bearing," Procedia Technology, vol. 14, no. November 2015, pp. 447-456, 2014.
[12] B. Corne, B. Vervisch, C. Debruyne, J. Knockaert, and J. Desmet, "Comparing MCSA with Vibration Analysis in order to detect Bearing Faults - A Case Study," pp. 1366-1372, 2015.

[13] M. Ahrens and L. Kucera, "Analytical calculation of fields, forces and losses of a radial magnetic bearing with a rotating rotor considering eddy currents," 5th Internat. Symp. on Magnetic Bearings, 1996.

[14] J. Hillyard, "Magnetic Bearings," no. April, pp. 1-43, 2006.

[15] K. Radman, N. Bulić, and W. Gruber, "Geometry Optimization of a Bearingless Flux- Switching Slice Motor,” pp. 1695-1701, 2015.

[16] P. Anantachaisilp and Z. Lin, "An experimental study on PID tuning methods for active magnetic bearing systems," vol. 5, no. 2, pp. $146-$ $154,2013$.

[17] A. Tenhunen, T. Benedetti, T. Holopainen, and A. Arkkio, "Electromagnetic forces in cage induction motors with rotor eccentricity," IEEE International Electric Machines and Drives Conference, vol. 3, no. June, pp. 1616-1622, 2003.

[18] D. G. Dorrell, J. K. H. Shek, and M. F. Hsieh, "The development of an indexing method for the comparison of unbalanced magnetic pull in electrical machines," IEEE Transactions on Industry Applications, vol. 2015, no. 1, pp. 145-153, 2015.

[19] G. Schweitzer, Magnetic Bearings, 1989.

[20] A. Chiba, T. Fukao, O. Ichikawa, M. Oshima, M. Takemo, and D. Dorrell, "Magnetic bearings and bearingless drive," Amsterdam, The Netherlands, 2005

\section{BIOGRAPHIES}

Bram Corne received his Master degree in Electrotechnical Engineering from the Technical University HoWest, Kortrijk, Belgium, in 2012. Since 2012 he has been a researcher regarding torque distortions due by voltage distortion on electrical machines. Since 2014 he is a Ph.D. student at the Ghent University, Ghent, Belgium, where he is researching the possibilities of a condition monitoring system with MCSA-technology for driven induction machines.

Jos Knockaert received the degree of industrial engineer in Electrotechnics in 1996 from the University College of Bruges-Ostend, the M.Sc. degree in Electronic System Design in 2001 from Leeds Metropolitan University and the Ph.D. degree in Engineering Science in 2009 from the K.U. Leuven. He worked as EMC-design engineer in the industry and became assistant at the University College of Bruges-Ostend. Since 2010, he is assistant professor at Ghent University, teaching electrical machines, electromagnetic compatibility and power electronics. He is member of the research group Lemcko with focus on electromagnetic compatibility, power electronics and high frequency problems in industrial systems.

Jan J. M. Desmet received the Polytechnical Engineer degree from the polytechnic academy in Kortrijk, the M.Sc. degree in Electrical Engineering from the V.U.Brussels and in his Ph.D. degree at the K.U.Leuven, Belgium. Currently he is full professor at the Ghent University teaching power quality, renewables and industrial electric measurement techniques. His research interests include LV distributed generation, renewable, power quality and their mutual interactions. Besides his job as full professor and researcher, he also is member of several expert colleges and both reviewer and topic chairman for international scientific conferences. He is also IEEE senior member, member of SC77A (IEC) and TC210 (CENELEC). His international scientific recognition is validated by his membership of the CIRED Technical Committee TC2 and his membership in the CIGRE JWG C4.24. His research lab is the only one over Europe with a free programmable artificial grid of 240kVA, including all renewable sources. Since November 2014, research group Lemcko became member of the DERLab group. 